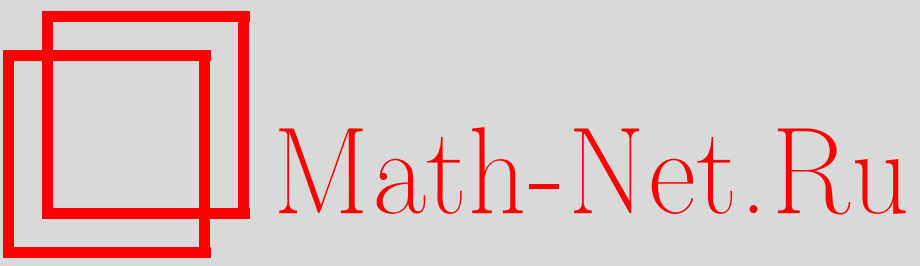

Б. С. Кругликов, Монотонность функции вращения и антисогласованные контактные структуры, УМH, 1996, том 51, выпуск 1, 153-154

DOI: https://doi.org/10.4213/rm929

Использование Общероссийского математического портала Math-Net.Ru подразумевает, что вы прочитали и согласны с пользовательским соглашением

http://www.mathnet.ru/rus/agreement

Параметры загрузки:

IP: 18.208 .226 .222

26 апреля 2023 г., 02:29:57 


\title{
МОНОТОННОСТЬ ФУНКЦИИ ВРАЩЕНИЯ И АНТИСОГЛАСОВАННЫЕ КОНТАКТНЫЕ СТРУКТУРЫ
}

\author{
Б. С. Кругликов
}

В [2], [4] (см. также [5]) построена теория траекторной классифиикации интегрируемых в боттовском смысле нерезонансных гамильтоновых систем с двумя степенями свободы. Системам с монотонными функциями вращения отвечают более простые траекторные инварианты. В этой заметке мы рассмотрим геометрию, отвечающую монотонности функции вращения.

Контактная структура $\xi$ на гиперповерхности $Q^{2 n-1}$ в симплектическом многообразии $\left(M^{2 n}, \omega\right)$ называется согласованной с симплектической формой $\omega$, если для локально задающей $\xi$ формы $\alpha$ выполнено условие $\left.\alpha \wedge \omega^{n-1}\right|_{Q} \neq 0$.

ОпредЕлЕниЕ 1 . Назовем функцию $\psi(t) k$-возмущенно-монотонной, если она представима в виде суммы $\psi(t)=\psi_{1}(t)+\psi_{2}(t)$, где функция $\psi_{1}$ монотонна, а возмушение $\psi_{2}$ не превосходит по абсолютной величине $k:\left|\psi_{2}(t)\right|<k$.

Рассмотрим нерезонансную боттовскую интегрируемую гамильтонову систему (ИГС) $v=\operatorname{sgrad}_{\omega} H$ на неособом изоэнергетическом многообразии $Q_{c}^{3}=\{H=c\} \subset\left(M^{4}, \omega\right)$, $d H(x) \neq 0$ при $x \in Q^{3}$. Дополнительный интеграл $F,\{H, F\}=0$, определяет гамильтоново действие $\mathbb{R}^{2}$ системы $v$ на $M^{4}$ (см. доказательство теоремы Лиувилля, [1]): точке $(t, s) \in \mathbb{R}^{2}$ соответствует сдвиг точек из $M^{4}$ за единичное время вдоль векторного поля $t \operatorname{sgrad}_{\omega} H+s \operatorname{sgrad}_{\omega} F$. Это действие можно рассматривать при ограничении на инвариантное многообразие $Q_{c}^{3}$. На любом ребре молекулы $e$ (см. [3]) - однопараметрическом семействе торов Лиувилля $T^{2} \times I-$ оно локально свободно. Так как на ребре все орбиты действия компактны, можно профакторизовать это действие до действия тора $T^{2}$, причем также гамильтонова. Действительно, в силу теоремы Лиувилля, на ребре существуют координаты $(\varphi, t)$ такие, что

$$
\left.\omega\right|_{e}=\left(\Phi_{1}(t) d \varphi^{1}+\Phi_{2}(t) d \varphi^{2}\right) \wedge d t
$$

Действие тора $T^{2}$ задается сдвигами вдоль $\partial_{\varphi^{1}}, \partial_{\varphi^{2}}$. Циклам $\left\{\varphi_{2}=\right.$ const $\},\left\{\varphi_{1}=\right.$ const $\}$ на торах Лиувилля $T^{2}(t)$ отвечают переменные действия $I_{1}, I_{2}$ такие, что $\operatorname{sgrad}_{\omega} I_{i}=\partial_{\varphi^{i}}$. Имеем: $v=v^{1}(t) \partial_{\varphi^{1}}+v^{2}(t) \partial_{\varphi^{2}}$.

ОПредЕЛЕниЕ 2. Функция $\rho(t)=v^{2}(t) / v^{1}(t), \rho: I \rightarrow \mathbb{R} \cup\{ \pm \infty\}$, называется функцией вращения данной ИГС на ребре $e . \psi=\operatorname{arcctg} \rho(t)(\bmod 2 \pi)$ называется угловой функцией. Знаки монотонности функций $\rho$ и $\psi$ противоположны.

Теорема 1 [6]. Для $T^{2} \times I \subset Q^{3} \subset\left(M^{4}, \omega\right)$ - ребра молекуль $W-$-ледующие свойства әквивалентны:

(1) существует контактная форма, согласованная с $\omega$ и инвариантная относительно гамильтонова действия $T^{2}$,

(2) угловая функция $\psi(t)$ является $\pi / 2$-возмущенно-монотонной.

СледствиЕ. Рассмотрим гамильтонову систему на $\left(T^{*} P^{2}, \omega=d p \wedge d q\right)$ с гамильтонианом $H=\frac{|p|^{2}}{2}+U(q)$, где $P^{2}-$ замкнутая поверхность, $(q, p)-$ стандартные координата-импульс. Если система интегрируема с боттовским интегралом, то при больиих әнергиях, $E \gg 1$, на любом ребре молекуль $W_{E}=W(Q=\{H=E\})$ угловая функция будет $\pi / 2$-возмущенно-монотонной.

ЗАмечАниЕ. Рассматриваемая гамильтонова система по принципу Мопертюи эквивалентна геодезическому потоку на двумерной поверхности. В случае известных интегрируемых геодезических потоков результат подтверждается явными формулами для функции вращения, полученными Е. Селивановой. 
ДокАЗАтельство. Рассмотрим стандартную форму $\alpha=p d q$ на $T^{*} P^{2}, d \alpha=\omega$; $d H \wedge \alpha \wedge d \alpha=d H \wedge \alpha \wedge \omega=(p d p+(\partial U / \partial q) d q) \wedge(p d q) \wedge(d p \wedge d q)=|p|^{2} d p_{1} \wedge d q^{1} \wedge d p_{2} \wedge d q^{2}$. Но при $E>\max _{q \in P^{2}} U(q)$ на $Q_{E}=\{H=E\}$ выполнено неравенство $|p| \neq 0$. Следовательно, $\left.\alpha \wedge d \alpha\right|_{Q}=\left.\alpha \wedge \omega\right|_{Q} \neq 0$ на $Q_{E}$ и $d H(x) \neq 0$ при $x \in Q$. Рассмотрим $T^{2} \times I$-произвольное ребро молекулы $W$. На нем задано гамильтоново действие группы $T^{2}$. Усредним $\alpha$ по этому действию:

$$
\alpha_{\mathrm{cp}}(w)=\frac{1}{(2 \pi)^{2}} \oint_{T^{2}} \alpha\left(\left.w\right|_{\varphi}\right) d \varphi^{1} \wedge d \varphi^{2} .
$$

Усредняя равенство $d \alpha=\omega$, получаем, в силу $\omega_{\mathrm{cp}}=\omega$, равенство $d \alpha_{\mathrm{cp}}=\omega$, а посколку усреднение формы объема суть форма объема, неравенство $\alpha \wedge \omega \neq 0$ влечет за собой неравенство $\alpha_{\mathrm{cp}} \wedge \omega \neq 0$. Теперь утверждение следует из теоремы.

Если же мы хотим получить условие монотонности, то $\alpha$ и $\omega$ надо связать более жестким условием, чем условие согласованности - условием антисогласованности $\alpha \wedge \omega=0$.

ТЕорема 2. Пусть $\left(Q,\left.\omega\right|_{Q}\right)$ - неособая изоэнергетическая поверхность боттовской ИГС $v=\operatorname{sgrad}_{\omega} H$ с интегралом $F$. На некотором ребре е угловая функиия монотонна тогда и только тогда, когда на нем существует такая инвариантная относительно гамильтонова действия тора $T^{2}$ контактная форма $\alpha$, что $\alpha \wedge \omega=0$.

Лемма 1. 1-форма а на ребре $T^{2} \times I \subset Q^{3}$ нерезонансной боттовской ИГС $v$ инвариантна относительно гамильтонова действия тора $T^{2}$, если и только если

$$
\left.\mathscr{L}_{v} \alpha\right|_{T^{2}} \equiv 0, \quad \mathscr{L}_{v} \mathscr{L}_{v} \alpha \equiv 0,
$$

где $\mathscr{L}_{v}$ - производная Ли, а $T^{2}=T^{2}(t)$ - лиувиллев тор ребра.

Теорема 3. Пусть $\left(Q^{3},\left.\omega\right|_{Q}\right)$ - неособая изоэнергетическая поверхность боттовской ИГС $v$. На ребре е угловая функчия монотонна тогда и только тогда, когда на нем существует трансверсальное лиувиллевым торам, инвариантное относительно гамильтонова действия $T^{2}$ векторное поле $\eta$, такое что $\omega\left(\eta, \mathscr{L}_{v} \eta\right) \neq 0$.

ЛЕмма 2. Векторное поле $\eta$ на ребре $T^{2} \times I \subset Q^{3}$ инвариантно относительно гамильтонова действия тора $T^{2}$, если и только если $\mathscr{L}_{v} \eta \in T\left(T^{2}\right)$ - касательное расслоение лиувиллевого тора, и $\mathscr{L}_{v} \mathscr{L}_{v} \eta \equiv 0$.

ЗАмечАниЕ. Можно считать функцию $f=\omega\left(\eta, \mathscr{L}_{v} \eta\right)$ заданной на ребре $e \ni t$. При изменении монотонности угловой функции $\psi=\psi(t)$, функция $f=f(t)$ меняет знак, а нули $f$ соответствуют экстремумам $\psi$.

Автор благодарен академику РАН профессору А. Т. Фоменко и д.ф.м.н. А.В. Болсинову за внимание к работе.

\section{СПИСОК ЛИТЕРАТУРЫ}

[1] Арнольд В.И. Математические методы классической механики. М.: Наука, 1989. [2] Болсинов А. В. // Матем. сб. 1995. Т. 186. № 1. С. 3-28. [3] Болсинов А. В., Матвеев С. В., Фоменко А. Т. // УМН. 1990. Т. 45. № 2. С. 59-77. [4] Болсинов А. В., Фоменко А. Т. // Матем. сб. 1994. Т. 185. № 4, 5. С. 27-80, 27-78. [5] Kruglikov B. Exact smooth classification of Hamiltonain systems on 2-manifolds // Preprint of ICTP, Trieste, Nov. 1994, IC/94/314. [6] Nguen Tien Zung. Symplectic topology of integrable Hamiltonian systems // Preprint of ICTP, Trieste. 1993, IC/93/305.

Московский государственный университет им. М. В. Ломоносова e-mail: borkru@difgeo.math.msu.su
Принято редколлегией 01.11 .1995 